3

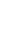
2 6

8

\title{
Systems Genetics of Single Nucleotide Polymorphisms at the Drosophila Obp56h Locus
}

\author{
Sneha S. Mokashi ${ }^{1}$, Vijay Shankar ${ }^{1}$, Joel A. Johnstun ${ }^{2}$, Wen Huang ${ }^{3}$ \\ Trudy F. C. Mackay ${ }^{1^{*}}$ and Robert R. H. Anholt ${ }^{1 *}$
}

1 Department of Genetics and Biochemistry and Center for Human Genetics, Clemson University, 114 Gregor Mendel Circle, Greenwood, SC 29646, USA

${ }^{2}$ Department of Biological Sciences, Program in Genetics, North Carolina State University, Raleigh, NC 27695, USA

${ }^{3}$ Department of Animal Science, Michigan State University, East Lansing, MI 48824, USA * Joint corresponding authors,

Address correspondence to Robert R. H. Anholt, e-mail: ranholt@clemson.edu or Trudy Mackay, email tmackay@clemson.edu

7 Running title: Drosophila Obp56h SNP Effects

Keywords: quantitative genetics, genotype-phenotype map, pleiotropy, fitness traits, RNA sequencing, micro-environmental variation 


\section{Abstract}

22 Variation in quantitative traits arises from naturally segregating alleles with environmentally

23 sensitive effects, but how individual variants in single genes affect the genotype-phenotype map

24 and molecular phenotypes is not understood. We used CRISPR/Cas9 germline gene editing to

25 generate naturally occurring variants with different site classes and allele frequencies in the

26 Drosophila melanogaster Obp56h gene in a common genetic background. Single base pair

27 changes caused large allele-specific and sexually dimorphic effects on the mean and micro-

28 environmental variance for multiple fitness-related traits and in the Obp56h co-regulated

29 transcriptome. However, these alleles were not associated with quantitative traits in the

30 Drosophila Genetic Reference Panel, suggesting that the small allelic effects observed in

31 genome wide association studies may be an artifact of averaging variable context-dependent

32 allelic effects over multiple genetic backgrounds. Thus, the traditional infinitesimal additive

33 model does not reflect the underlying biology of quantitative traits. 


\section{Main}

35 Quantitative traits vary continuously in natural populations due to segregating alleles at many genes with environmentally sensitive effects ${ }^{1,2}$. Understanding the genetic and environmental basis of variation for quantitative traits is important for precision medicine, agriculture, and evolutionary biology. However, it is challenging to dissect the genotype-phenotype map at base pair resolution because quantitative trait locus mapping studies are limited in precision due to blocks of linkage disequilibrium (LD) in linkage and association mapping populations, within which molecular polymorphisms are not independent; and effects of individual rare variants cannot be evaluated using association mapping. In addition, most molecular polymorphisms associated with quantitative traits are in non-coding genomic regions and presumably affect complex organismal phenotypes via regulation of gene expression, not only of the gene most proximal to the variant, but also of co-regulated genes ${ }^{3-6}$. There is also a growing realization that naturally occurring polymorphisms can be associated with micro-environmental variance as well as mean values of quantitative traits; i.e., the within-genotype phenotypic variance can differ between alternative alleles ${ }^{7-15}$.

Here, we used a CRISPR/Cas9 mediated gene deletion and reinsertion strategy to generate an

51 allelic series of closely linked single nucleotide polymorphisms (SNPs) in a 738 base pair region including the Drosophila melanogaster Odorant binding protein 56h (Obp56h) gene. Obp56h is an excellent candidate for CRISPR/Cas9 germline gene editing since it is a member of a multigene family ${ }^{16-18}$ for which functional redundancy is likely to prevent lethality upon gene deletion; it is a small gene (651 bp) without nested genes; and the nearest genes are 12,891 bp upstream and 10,374 bp downstream. There is also evidence that Obp genes have pleiotropic

57 effects on quantitative traits. Other members of the Obp gene family have pleiotropic functions 58 that extend beyond their traditional roles in chemosensation ${ }^{4,19-21}$. RNA interference of Obp56h affects olfactory behavior ${ }^{22}$, avoidance of bitter tastants ${ }^{23}$, mating behavior ${ }^{24}$, and expression of 
co-regulated genes associated with lipid metabolism, immune/defense response, and heat

61 stress $^{24}$. Obp56h is expressed in chemosensory tissues ${ }^{25}$ and in the central brain ${ }^{26,27}$, which

62 suggests functional pleiotropy at the Obp56h locus.

63

64 We generated an Obp56h null allele by inserting a transgene with a selectable marker in the 65 endogenous Obp56h genomic location, and then excised the selectable marker and replaced it 66 with transgenes containing the minor allele for each of five Obp56h SNPs that segregate in the 67 D. melanogaster Genetic Reference Panel (DGRP) ${ }^{28,29}$, all in a common genetic background. 68 Three of the SNPs are common, with minor allele frequencies (MAF) $\geq 0.05$, and two are rare 69 (MAF < 0.05); two are protein coding missense polymorphisms and three are potentially regulatory variants located upstream and downstream of the gene body and in the 3' UTR (Fig. 1). We quantified the effects of each SNP on the mean and micro-environmental variance of multiple fitness-related quantitative traits and on the transcriptome. This enabled us to compare

73 the pleiotropic effects of multiple SNPs in one gene that are in LD in a natural population and of rare vs. common, protein coding vs. noncoding variants on organismal quantitative traits and the co-regulated transcriptome. We found extensive functional pleiotropy of Obp56h, and heterogeneous, large, and sexually dimorphic allelic effects for all organismal and transcriptional

77 phenotypes. This reverse genetic engineering strategy can be generally applied to other genes

78 to dissect variation in the genotype-phenotype relationship at single base pair resolution.

\section{Results}

\section{Generation of Obp56h allelic series}

82 There are a total of 104 SNPs and 16 insertion/deletion polymorphisms in the 2,651 bp genomic 83 region including the Obp56h locus and $1 \mathrm{~kb}$ up- and down-stream of this locus in the DGRP. We 84 selected five SNPs with MAFs ranging from $0.006-0.26$ : Obp56h ${ }^{A 5510 C}$ is $44 \mathrm{bp}$ upstream of the 
annotated transcription start site; the minor alleles of Obp56h $h^{T 5613 C}$ and $O b p 56 h^{C 5849 G}$ are missense mutations in the first and second exon, respectively; $O b p 56 h^{A 6182 T}$ is in the $3^{\prime}$ UTR; and Obp56h $h^{T 6247 A}$ is 43 bp downstream of the annotated end site (Fig. 1; Table 1). The MAF of Obp56h $h^{C 5849 G}$ and Obp56h $h^{A 6182 T}$ are $<0.05$ in the DGRP; these polymorphisms are underpowered for genome-wide association analyses. The SNP names begin with the common allele variant and end with the minor allele variant; the four intervening numbers are the last four digits of the genomic location. Allele names are the genomic locations followed by the nucleotide. Although LD declines rapidly with physical distance in $D$. melanogaster ${ }^{28,29}$, these SNPs are in strong LD in the DGRP and therefore not independent (Supplementary Table 1).

We designed a two-step strategy to assess the effects of individual SNPs on organismal phenotypes and the Obp56h co-regulated transcriptome. First, we used CRISPR/Cas9 germline gene editing to generate a deletion of the Obp56h gene, substituting instead a DsRed fluorescent marker. This null allele is designated Obp56h: In the second step, we inserted Obp56h genomic sequences that contain the minor allele of each of the five SNPs in the endogenous location to generate an Obp56h allelic series in a common genetic background (CSB, Fig. 1; Supplementary Fig. 1; Supplementary Table 2). The CSB allele has the common allele for each of the five SNPs. All transgenes were verified by Sanger sequencing.

\section{Effects of Obp56h alleles on organismal phenotypes}

We assessed the effects of Obp56h null and SNP alleles on the mean values of several fitnessrelated traits: viability, sex ratio, feeding behavior, starvation stress resistance, time to recover from a chill-induced coma, heat stress resistance, locomotor activity, and sleep traits. All variants had reduced viability relative to the CSB control, with a greater effect for the SNP minor alleles $(P<0.0001)$ than the null allele $(P<0.05)($ Fig. 2A; Supplementary Table 3$)$. To assess whether effects on viability were different for male and female offspring, we calculated sex ratios 
111 and observed that the average number of eclosing males was less than the number of females

112 for the Obp56h $h^{5613 C}, O b p 56 h^{5849 G}, O b p 56 h^{6182 T}$ and Obp56h $h^{6247 A}$ alleles (Fig. 2B; Supplementary

113 Table 3).

115 The Obp56h alleles had heterogeneous, often sexually dimorphic effects on all other

116 quantitative traits. Obp56h females (but not males) consumed less sucrose than the CSB

117 control $(P<0.05)$. The Obp56h $h^{5613 C}$ allele had the strongest effect on consumption levels, with

118 both males $(P=0.0064)$ and females $(P<0.0001)$ drinking significantly less than the control

119 (Fig. 2C and 2D; Supplementary Table 3). The Obp56h ${ }^{5510 C}$ allele had a male-specific increase

120 in sucrose consumption $(P=0.029)$; and $\operatorname{Obp56h}^{5849 G}(P<0.0001)$, Obp56h $^{6182 T}(P<0.0001)$

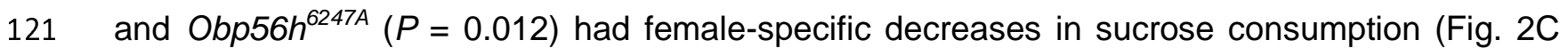

122 and 2D; Supplementary Table 3).

124 Obp56h females $(P<0.0001)$, but not males, were more resistant to starvation stress than the control. All SNP minor alleles showed increased survival time under starvation conditions than

126 the major allele in females. In males, alleles of Obp56h $h^{5510 C}, O b p 56 h^{5613 C}$ and Obp56h ${ }^{5849 G}$ had

127 increased survival time under starvation stress; Obp56h $6182 T$ and Obp56h ${ }^{6247 A}$ were not

128 significantly different from CSB (Fig. 2E, 2F, 2G, 2H; Supplementary Table 3). With respect to

129 time to recovery from a chill-induced coma, the Obp56h allele slightly decreased recovery time

130 (i.e., in the direction of increased fitness) in females only $(P=0.03)$, while the only SNP to affect

131 chill coma recovery time was Obp56h ${ }^{T 6247 A}$, for which the minor allele increased recovery time in

132 females $(P<0.0001)$ and males $(P=0.0006)$ (Fig. 2 I and 2J; Supplementary Table 3). The

133 most heterogeneous effects of Obp56h alleles we observed were for survival following heat

134 stress. Obp56h males $(P<0.0001)$, but not females, had increased survival compared to CSB.

135 However, Obp56h $h^{5613 C}$ had markedly increased survival following heat stress in males $(P<$ $1360.0001)$ and females $(P<0.0001)$; Obp56h $h^{5849 G}$ was not significantly different from CSB in either 
137

138

139

140

141

142

143

144

145

146

147

148

149

150

151

152

153

154

155

156

157

158

159

160

sex; Obp56h ${ }^{5510 C}$ had a female-specific increase in survival time after heat stress $(P=0.0007)$; and $O b p 56 h^{6182 T}$ and $O b p 56 h^{6247 A}$ had increased survival times but with smaller effects than Obp56h ${ }^{5613 C}$ (Fig. 2K and 2L; Supplementary Table 3).

Obp56h $h^{5510 C}$ did not significantly affect total locomotor activity in either sex, but activity was increased in males for all other alleles and for Obp56h $h^{5613 C}$ and $O b p 56 h^{5849 G}$ in females (Fig. 3A and 3B; Supplementary Table 3; the activity of $O b p 56 h^{-}$females decreased compared to CSB (Fig. 3B; Supplementary Table 3). In females, the proportion of time spent sleeping during the night was increased relative to CSB for Obp56h and minor alleles of all SNPs. Day sleep in females was similarly increased for all but $O b p 56 h^{5510 C}$, which was not significantly different from CSB. In contrast, only Obp56h ${ }^{6182 T}$ affected night sleep in males. Obp56h and Obp56h $h^{5510 C}, O b p 56 h^{5613 C}$ and Obp56h $h^{6247 A}$ had increased day sleep in males (Fig. 3C and 3D; Supplementary Table 3).

In summary, single Obp56h SNPs have pleiotropic and sexually dimorphic effects on the mean values of all organismal quantitative traits we assessed (Supplementary Table 4). The effects of the common SNP and minor SNP alleles for each trait are heterogeneous, ranging from large to not significantly different from each other. Many of the alleles exhibit fitness trade-offs; for example, trade-offs between reduced viability and increased resistance to starvation and heat stress and between increased resistance to heat stress but a longer time to recover from chill coma stress. The SNP alleles typically have effects in the same direction as the null allele, but the SNP allele effects are often greater than the null allele effects. 
161 We previously evaluated the effects of the Obp56h SNPs for a subset of the organismal

162 quantitative traits evaluated in this study using genome wide association analyses in the

163 DGRP ${ }^{11,12,29,30}$. This affords us the opportunity to compare the effects of the same homozygous

164 SNPs in a common genetic background vs. averaged over multiple genetic backgrounds. In

165 contrast to the large and significant SNP effects observed in CSB, the effects were small and

166 not significant in the DGRP (Supplementary Table 5). This observation is inconsistent with

167 independent additive SNP effects and implies the existence of epistatic modifier loci in the

168 DGRP that on average suppress the effects of the Obp56h SNPs on organismal phenotypes

169 and/or variants in linkage disequilibrium with the Obp56h SNPs that counter their effects.

Effects of Obp56h alleles on micro-environmental variance of organismal phenotypes

172 Micro-environmental variance (or general environmental variance ${ }^{1}$ ), refers to the phenotypic

173 variation for a quantitative trait that occurs among individuals of the same genotype when they

174 are reared in a common environment. We performed formal analyses of variance of micro-

175 environmental variance for Obp56h alleles (Supplementary Table 4) and found that this

176 phenomenon is pervasive: the micro-environmental variance for all alleles is significantly

177 different from that of CSB for multiple organismal phenotypes. Changes in micro-environmental

178 variance are allele-specific within each trait and are often sex-specific for each allele. The

179 pleiotropic effects of Obp56h alleles on micro-environmental variance vary by trait and allele;

180 e.g., micro-environmental variance is largely increased for heat shock survival and largely 181 decreased for sleep traits. Effects of Obp56h alleles on micro-environmental variance are 182 decoupled from their effects on trait means: most alleles affect either the mean or the micro183 environmental variance for any sex/trait combination, although some alleles affect both mean 184 and the micro-environmental variance in the same or opposite directions for a given sex and 185 trait (Supplementary Table 4). 
187 Effects of Obp56h alleles on genome wide gene expression

188 To investigate the cellular processes that might underlie the observed sexually dimorphic 189 pleiotropic effects of Obp56h alleles, we obtained whole transcriptome profiles for heads from males and females separately, and identified differentially expressed genes among the CSB, Obp56h and Obp56h SNP minor alleles (Supplementary Tables 6 and 7). Obp56h expression is obliterated in both sexes in the Obp56h null allele compared to CSB and is partially restored in the reinsertion lines (Fig. 4A). At a false discovery rate (FDR) $<0.05$, we identified 1,009 (717) differentially expressed genes in females (males) in any comparison between two alleles (Supplementary Table 7). A total of 406 co-regulated genes are in common between males and females, 603 are female-specific and 311 are male-specific. Gene set enrichment analyses ${ }^{31}$ reveal that differentially expressed genes in common between males and females and malespecific genes are enriched for terms involved in mitochondrial function, whereas genes that are only differentially expressed in females are enriched for terms involving protein translation, transport and localization, development and signal transduction (Supplementary Table 7).

Pairwise comparisons between the genotypes for females and males separately show that the number of coregulated differentially expressed genes varies greatly among alleles within each sex and between sexes for each pair of alleles (Fig 4B). However, in general the majority of the co-regulated genes have increased expression in Obp56h ${ }^{5510 C}, O b p 56 h^{5613 C}, O b p 56 h^{6182 T}$ and $206 O b p 56 h^{6247 A}$ relative to $C S B$ and Obp56h in both sexes; while the same genes have decreased expression in $O b p 56 h^{5849 G}$ relative to $C S B$ in males and females (Supplementary Table 8). This pattern is reversed for a second, smaller group of co-regulated genes (Supplementary Table 8).

We mapped the genes encoding differentially expressed transcripts onto known protein-protein 211 interaction networks, separately for males (Fig. 5) and females (Fig. 6). The large network in 
212 each sex could be partitioned and clustered into smaller subnetworks that functionally converge

213 toward oxidative phosphorylation, mitochondrial translation, circadian cycle, glutathione

214 metabolism, ubiquitin-dependent proteolysis and cellular response to starvation in males (Fig. 5)

215 and cytoplasmic translation, protein modification and localization, regulation of transport, G-

216 protein coupled receptor signaling, mRNA splicing, chitin development and histone acetylation

217 in females (Fig. 6). Both male and the female networks contained a large subnetwork that was

218 enriched for electron transport chain and oxidative phosphorylation (Figs. 5 and 6).

221 We computed the coefficients of variation ( $C V$, standard deviation/mean) across the replicates

222 from normalized expression counts for each Obp56h SNP minor allele as well as for CSB and

223 the Obp56h null allele. We plotted the distributions of $C V$ across all expressed genes and all

224 genotypes, separately in males and females (Supplementary Fig. 2). The distributions of CV

225 were highly right-skewed; therefore, we chose genes with median expression above 10 counts

226 per million across all alleles, for which at least one allele had a $C V \geq 1$ and for which the

227 variance heterogeneity analysis across alleles from Levene's test had an FDR $<0.05$ as

228 contributing significantly to transcriptional micro-environmental plasticity. A total of 246 genes in

229 males and 71 genes in females met these criteria for at least one variant. Obp56h $h^{6247 A}$ and

$230 O b p 56 h^{6182 T}$ had the largest number of transcripts with high micro-environmental plasticity in

231 both sexes, and Obp56h had the smallest number of high plasticity transcripts in males and

232 second smallest in females (Supplementary Table 9). Multi-dimensional scaling (MDS) based

233 on the $C V$ values showed that $O b p 56 h^{6247 A}$ and $O b p 56 h^{6182 T}$ were separated from the other

234 alleles, but on different axes, indicating that the transcripts associated with high $C V$ were

235 different for these alleles (Supplementary Fig. 3). A total of 36 (50.7\%) of the gene affecting

236 micro-environmental plasticity of the transcriptome in females overlapped the genes associated

237 with transcriptional micro-environmental plasticity in males. However, there was very little 
238

239

240

241

242

243

244

overlap between genes affecting the mean and micro-environmental plasticity of transcript abundances in either sex.

Gene Ontology (GO) enrichment analyses also showed distinct enrichment categories for $O b p 56 h^{6247 A}$ and $O b p 56 h^{6182 T}$. Transcripts with high micro-environmental plasticity in $O b p 56 h^{6247 A}$ males are enriched for terms involving immune response, response to stress, and lipase activity; whereas in Obp56 $h^{6182 T}$ males the enrichment was for transcripts associated with RNA binding, Box C/D RNP complex and the spliceosomal complex (Supplementary Table 9). Similar differences in enriched GO categories were observed in females but to a lesser extent due to the smaller number of transcripts with high micro-environmental plasticity (Supplementary Table 9). In addition to protein coding genes, regulatory non-coding transcripts contribute to micro-environmental variation in the transcriptome, especially snoRNAs and tRNAs (Supplementary Table 9).

\section{Discussion}

The genetic architecture of quantitative traits inferred from many linkage mapping and genome wide association analyses in humans ${ }^{32-34}$ and model organisms, including Drosophila ${ }^{35-37}$, is highly polygenic, with large numbers of genes each with small additive effects, consistent with the infinitesimal model proposed by Fisher over a century ago ${ }^{38}$. The small effects could be because of imperfect LD between the genotyped variant and the true causal variant ${ }^{32,39}$, because the effects are truly small in all genetic backgrounds, or because the allelic effects are highly context-dependent and vary according to sex, environmental conditions and genetic background such that marginal (additive) effects over all contexts are small ${ }^{40}$. These possibilities can only be distinguished by examining the effects of naturally occurring SNPs in a common genetic background, which is now feasible using advanced germline gene editing technology ${ }^{41,42}$. 
We found large effects of five naturally occurring SNPs in Obp56h on a battery of fitness-related organismal quantitative traits. The effects of the five SNPs varied in magnitude, and

267 occasionally direction, within each trait, and the pattern of pleiotropic effects varied across traits.

268 All alleles had sex-specific effects on at least one trait, but the pattern of sex-specificity was 269 different for each trait/allele combination. There was no difference in the numbers of significant 270 effects between rare and common variants $(P=0.64$, Fisher's Exact Test), as is typically found 271 in genome wide association analyses ${ }^{32,37}$; nor between missense and regulatory variants $(P=$ 272 0.49, Fisher's Exact Test). We observed fitness trade-offs at the single variant level, which 273 imposes an evolutionary constraint on natural selection at this locus (in the CSB genetic background). The observations that SNP allele effects are usually greater than those of the null 275 allele and that the effects of the same alleles on the same traits measured in the DGRP were small and not significant are both consistent with genetic background effects (epistasis). The

277 phenotypic effects of reduced expression of genes via RNA interference are often greater than 278 those of null alleles ${ }^{43-45}$, thought to be due to a compensatory mechanism induced only by the 279 null allele. Naturally occurring variants in the DGRP suppress the effects of new mutations ${ }^{46,47}$ and associations of DGRP alleles with quantitative traits vary according to population allele 281 frequency, a hallmark of epistasis ${ }^{24,40,48-50}$. The naturally occurring Obp56h variants also affect 282 the micro-environmental variance of multiple organismal quantitative traits. Together, all of 283 these observations suggest that the small effects of alleles affecting quantitative traits in 284 genome wide association studies are the consequence of averaging over multiple genetic 285 backgrounds, males and females, and environmental contexts, and that effects in any one 286 context may well be large. Although the infinitesimal statistical model fits these data, it 287 obfuscates the underlying biology. 
Most variants associated with quantitative trait variation are non-protein coding and could thus affect traits by perturbing expression of large genetic regulatory networks in relevant cell types, in the same way that a mutation in a single gene affecting a complex trait has quantitative 292 effects on the abundance of many co-regulated transcripts ${ }^{3,51,52}$, called the transcriptional niche 293 of the focal gene $e^{6,21}$. This concept is related to the omnigenic model of quantitative genetics ${ }^{5}$, 294 which postulates that gene regulatory networks are highly interconnected, such that any variant 295 in a core gene affecting a particular phenotype expressed in cell types relevant to the phenotype 296 will affect many co-regulated genes. These concepts provide possible molecular bases for the highly polygenic, pleiotropic genetic architecture of quantitative traits.

299 This study provides support for these models for naturally occurring SNPs. Different variants in the Obp56h core gene have widespread trans effects on the transcriptome that are sex-specific and partially shared and partially distinct among the different alleles. Missense variants as well as variants in non-coding regions impact the Obp56h transcriptional niche; the largest number of co-regulated genes in both sexes is for $O b p 56 h^{5849 G}$, a rare missense variant. The enrichment of co-regulated genes involved in mitochondrial function provides a functional explanation for the sex-specific, pleiotropic effects of Obp56h variants on viability, food consumption, stress resistance, activity and sleep traits. Most Obp56h minor alleles affect increased transcription of mitochondrial genes, consistent with increased starvation and heat stress resistance, and increased activity and sleep duration. However, the correspondence between transcriptional coregulation and organismal phenotypes is not perfect. The Obp56h ${ }^{5849 G}$ allele has decreased expression of co-regulated genes relative to the other alleles, but the direction of the effects on

311 organismal phenotypes is the same as for the other alleles, suggesting additional information 312 than transcriptional co-regulation will be needed to predict effects on organismal phenotypes. 
314 The Obp56h alleles also affect the micro-environmental plasticity of the transcriptome

315 independent of the allelic effects on mean transcript abundance, providing a functional

316 explanation for micro-environmental plasticity for organismal phenotypes. However, the

$317 O b p 56 h^{6182 T}$ and Obp56h $h^{6247 A}$ alleles, which have the largest number of transcripts with

318 significant micro-environmental variance, are not different from the other alleles in terms of

319 micro-environmental variance of organismal phenotypes, suggesting additional mechanisms

320 buffer the transcriptome - organismal phenotype relationship.

321

322 We chose Obp56h for its favorable properties for CRISPR/Cas9 gene editing and because 323 previous studies suggested that this gene might have pleiotropic effects on the transcriptome 324 and organismal phenotypes ${ }^{22-24}$. Members of the Obp gene family have been implicated in 325 chemosensation as carriers of hydrophobic odorants ${ }^{53,54}$. In that context, there is differential expression of six other Obp genes (Obp56g, Obp57a, Obp57b, Obp57c, Obp99b, Obp99c) in

327 males. Our results give further insight regarding the roles of Obp genes in additional nonchemosensory phenotypes ${ }^{4,21,24}$. Obp56h is expressed in the antenna and labellum ${ }^{25}$ and in cells of the central brain ${ }^{26,27}$. The ligand(s) for Obp56h in the brain are not known, but could be hydrophobic metabolites, which play a role in fundamental cellular processes that include mitochondrial metabolism and RNA processing. The extent to which naturally occurring 332 polymorphisms affect these processes may lead to pleiotropic fitness phenotypes with different 333 effects in males and females.

\section{Methods}

336 Generation of transgenic lines

337 The protocols used to generate the Obp56h deletion and allelic reinsertions are similar to those 338 described previously ${ }^{21,55,56}$. Primer details are given in Supplementary Table 1. To generate a 
CRISPR/Cas9 mediated null allele of Obp56h in a CSB genetic background we designed two

340 guide RNAs flanking the gene using the Optimal Target Finder online tool ${ }^{55}$ and cloned them

341 into the pU6-Bbs1-chiRNA plasmid. We used the pBS-Hsp70-Cas9 plasmid as a source for

342 Cas9 and generated a donor plasmid containing 3XP3-driven DsRed flanked by $1 \mathrm{~kb}$ sequences

343 homologous to the regions flanking the Obp56h gene. This vector also contains loxP sites

344 flanking the DsRed cassette for subsequent removal of the cassette, and an attB site for site-

345 specific PhiC31 recombination to generate the reinsertion lines. We then generated the

346 reinsertion alleles from the Obp56h deletion ${ }^{21}$. We generated allelic variants of Obp56h via site-

347 directed mutagenesis in a pattB vector, which contained the CSB variant of the Obp56h gene.

348 To generate an Obp56h allelic series, plasmids were injected into Obp56h knockout fly embryos

349 (Model System Injections, Durham, NC).

351 Fly husbandry

352 We reared all flies at $25^{\circ} \mathrm{C}, 60-75 \%$ relative humidity and 12 -hr light-dark cycle on standard 353 cornmeal-molasses-agar medium. Prior to experimentation, we reared the flies for two 354 generations at controlled densities (5 males and 5 females per vial allowed to lay eggs for 2 days). We used 3-5-day old flies for all experiments.

\section{Organismal phenotypes}

358 Viability and sex ratio: We placed 25 males and 25 females into egg collection cages with grape

359 juice agar. We allowed the flies to acclimatize for $24 \mathrm{hr}$, with grape plate changes every $12 \mathrm{hr}$.

360 After that, we changed the plates every $12 \mathrm{hr}$ and collected 50 eggs per vial using a blunt

361 moistened micro-probe and placed them in vials with standard culture medium. We scored the

362 number and sex of flies that emerged until all pupae had eclosed from each of 10 vials per 363 genotype. 
Sucrose consumption: We performed capillary feeding (CAFÉ) assays as described

365

366

367

368

369

previously ${ }^{12,57}$ with a single fly per vial. We scored a minimum of 18 flies per genotype and sex. Starvation stress resistance: We used Drosophila Activity Monitors to measure starvation stress resistance. We placed one fly per tube containing starvation medium $(1.5 \%$ agar in distilled water) and ran the assay for 4 days in accordance with previous work ${ }^{58}$, with a total of 64 flies per sex per genotype. We obtained activity bout data using Shiny-R DAM ${ }^{59}$ and used the time of last activity bout as the time of death.

Recovery from chill-induced coma: We modified the original protocol for chill coma recovery assessment $^{60}$ to enable us to measure accurately timepoints of recovery for 20 flies simultaneously by recording videos of the recovery period. For each genotype, we sorted 20 flies per vial, sexes separately, 4 replicates, into vials with $2 \mathrm{ml}$ food the evening before the assay. On the morning of the assay, we transferred the flies to empty vials and placed them in an ice bucket filled with wet ice for $3 \mathrm{hr}$. The ice-anesthesia 3-hr periods were staggered for the genotypes to be assessed on an assay day to allow us to record videos for approximately 30 minutes per vial. We gently placed the flies from the ice into wells of a 24 -well microtiter plate with 2-5 flies per well for observation on an LED light box (Amazon) under a video camera (Canon). We recorded the flies for 30 min to determine how long it takes for each fly to right itself.

Response to heat shock: The day before measuring the response to heat shock, flies of each genotype were lightly anesthetized with $\mathrm{CO}_{2}$ and sorted in single-sex groups of 20 individuals in standard vials containing $5 \mathrm{ml}$ food. On the day of the heat stress exposure, flies from each replicate vial were transferred without anesthesia into vials without food and placed in an incubator at $37^{\circ} \mathrm{C}\left( \pm 0.5^{\circ} \mathrm{C}\right)$ for $180 \mathrm{~min}$. After heat stress exposure, flies were immediately transferred to vials containing $5 \mathrm{ml}$ of standard cornmeal-agar-molasses medium and returned to the $25^{\circ} \mathrm{C}$ incubator for $24 \mathrm{~h}$. The percentage of surviving flies per vial was recorded $24 \mathrm{~h}$ after 
the $3 \mathrm{hr}$ heat shock. A fly was considered alive if it could move when the vial was gently tapped. We performed five replicates per genotype and sex.

Activity and sleep: We assessed total activity and proportion of sleep during the day and night ${ }^{61,62}$ using Drosophila Activity Monitors (TriKinetics). We ran the assay in accordance with previously published work ${ }^{58}$ and recorded data for 5 days on at least 64 flies per sex per genotype. We processed the initial data using Shiny-R DAM ${ }^{59}$.

395 Statistical analyses: For phenotypes for which measurements were obtained for both sexes, we 396 assessed mean differences among the genotypes using factorial, fixed effects ANOVA models 397 for all seven genotypes: $Y=\mu+$ Genotype $+\operatorname{Sex}+$ Genotypex $\operatorname{Sex}+\varepsilon$, where $Y$ is the 398 phenotype, $\mu$ is the overall mean and $\varepsilon$ is the residual (error) variance. For viability and sex 399 ratio, we ran the reduced ANOVAs $Y=\mu+$ Genotype $+\varepsilon$. We also performed $t$-tests to identify 400 the genotypes which were significantly different from the CSB control (planned comparisons). 401 All analyses were performed using SAS Studio release 3.71 (SAS Institute, Cary, NC). To assess micro-environmental variance, we performed Levene's and Brown- Forsythe tests of heterogeneity of within line variance, separately for males and females ${ }^{13}$ for all seven genotypes, and for pairwise comparisons between Obp56h alleles and CSB.

\section{RNA sequencing}

407 To prepare libraries for RNA sequencing we collected 3-4 replicates of 50 flies, sexes 408 separately, between $1 \mathrm{pm}$ and $3 \mathrm{pm}$ and flash froze them on dry ice in $15 \mathrm{ml}$ Falcon tubes 409 (Thermo Fisher Scientific, Waltham, MA). The flies were decapitated using a strainer (Carolina 410 Biological Supply Company, Burlington, NC) for head collections ${ }^{63}$. The heads were collected on 411 a dry ice-cooled fly pad and placed in $2 \mathrm{ml}$ pre-filled tough microfuge tubes with glass beads.

412 Total RNA was extracted using the Direct-Zol microprep kit RNA extraction protocol (Zymo 413 Research, Irvine, CA). The heads were homogenized in a bead mill (Thermofisher) for $1 \mathrm{~min}$ at 
$4144 \mathrm{~m} / \mathrm{s}$, after which the RNA was eluted with $15 \mu \mathrm{L}$ water. We depleted ribosomal RNA using the

415 NuQuant +UDI, Drosophila AnyDeplete kit (Tecan, Männedorf, Switzerland) and prepared bar-

416 coded cDNA libraries for sequencing on an S1 flow cell on the NovaSeq 6000 platform (Illumina,

417 San Diego, CA) as described previously ${ }^{21}$.

\section{Analysis of RNA sequences}

420 We performed the initial steps of raw read processing and normalization of expression as

421 previously described ${ }^{21}$. Briefly, we used the AfterQC pipeline ${ }^{64}$ to trim adapters, detect abnormal

422 polynucleotide sequences, filter for low quality $(\mathrm{Q} \square<\square 20)$ and short (<35 nt) sequence reads

423 and generate of basic sequence quality metrics. We used the bbduk command from the

424 BBTools package ${ }^{65}$ to detect rRNA contamination. We aligned high-quality sequence reads to

425 the Drosophila melanogaster reference genome release 6 (version 6.13) using GSNAP aligner ${ }^{66}$

426 and mapped unique alignments to genes using the Subread package ${ }^{67}$. We excluded genes

427 with fewer than $25 \%$ nonzero read counts or a median count of $<2$ from further analyses. We

428 used $\mathrm{GeTMM}^{68}$ to normalize filtered expression counts. We ran ANOVAs across all seven

429 genotypes $(Y=\mu+$ Genotype $+\varepsilon)$ separately for males and females for each expressed

430 transcript using PROC GLM in SAS Studio release 3.71 (SAS Institute, Cary, NC) to identify

431 genes with significant (Benjamini-Hochberg FDR < 0.05) differential expression. We ran

432 individual contrast statements for pairwise comparisons and then filtered them to only include

433 genes that passed FDR in the overall model. We performed Gene Ontology analysis by

434 statistical overrepresentation tests using PantherDB ${ }^{31}$. We generated protein-protein interaction

435 networks from all differentially expressed genes (sexes separately) using the StringApp plugin

436 of Cytoscape 3.8.2 followed by $\mathrm{MCODE}^{69}$ analysis to identify clusters of subnetworks.

437 Functional annotation of the subnetworks was accomplished by performing Gene Ontology 
enrichment analysis on the membership. Labels were derived from GO biological processes with statistically significant enrichment (Benjamini-Hochberg FDR < 0.05).

Analysis of transcriptional micro-environmental plasticity was performed by first calculating the

442 coefficient of variation ( $\mathrm{CV}$ ) across the replicates for each allele, separately for males and

443 females, which showed that genes for which $C V \geq 1$ were in the extreme right tail of the

444 distribution. We also determined FDR values for Levene's test of variance heterogeneity for

445 estimates of between-replicate variance across all genotypes for each expressed gene, 446 separately for males and females. Significant genes for transcriptional micro-environmental 447 plasticity were those for which $C V \geq 1$ for at least one allele, Levene's test FDR for the gene < $448 \quad 0.05$; and median normalized expression across all genotypes was 10 or greater counts per 449 million. Multivariate ordination analysis was performed on the $C V$ values of these genes for 450 males and females separately using the cmdscale function that is part of the stats package in R.

451 We also performed Gene Ontology enrichment analyses by allele and overall for co-regulated 452 genes passing these criteria, separately for males and females.

454 Acknowledgements

455 We thank Dr. Lakshmi Sunkara for help with RNA sequencing and Rachel C. Hannah for 456 technical assistance with behavioral assays. This work was supported by NIH grant 1R01 457 GM128974 to TFCM and RRHA.

Data availability

460 RNA sequence data have been deposited in the GEO data repository under accession number

461 GSE178635. All code is available at

462 https://github.com/snehamokashi/Systems_genetics_of_SNPs_at_Obp56h 


\section{Author Contributions}

465 SSM generated the Obp56h allelic series and performed all experiments; SSM and VS 466 performed analyses on Obp56h differentially co-regulated transcripts; JAJ made Obp56h; WH

467 analyzed DGRP data; TFCM and RRHA conceptualized and directed the research program and 468 provided resources; SSM, VS, TFCM and RRHA wrote the manuscript.

469

\section{$470 \quad$ Competing Interest Statement}

471 The authors declare no competing interests.

472

\section{References}

474 1. Falconer, D. S. \& Mackay, T. F. C. Introduction to Quantitative Genetics. 4/e. Iongman, 475 Harlow, Essex (1996).

476

2. Lynch, M. \& Walsh,

B. Genetics and Analysis of Quantitative Traits. Sinauer, Sunderland, MA (1998).

478 3. Anholt, R. R. H. et al. The genetic architecture of odor-guided behavior in Drosophila: epistasis and the transcriptome. Nat. Genet. 35,180-184 (2003).

4. Arya, G.H. et al. Natural variation, functional pleiotropy and transcriptional networks (2010).

483 5. Boyle, E. A., Li, Y. I. \& Pritchard, J. K. An expanded view of complex traits: From polygenic to omnigenic. Cell 169, 1177-1186 (2017).

485 6. Anholt, R. R. H. \& Mackay, T. F. C. The road less traveled: from genotype to phenotype in flies and humans. Mamm. Genome 29, 5-23 (2018). 
487

7. San Cristobal-Gaudy, M., Bodin, L., Elsen, J. M. \& Chevalet, C. Genetic components of

505

506 litter size variability in sheep. Genet. Sel. Evol. 33, 249-271 (2001).

8. Sorensen, D. \& Waagepetersen, R. Normal linear models with genetically structured residual variance heterogeneity: a case study. Genet. Res. 82, 207-222 (2003).

9. Mackay, T. F. C. \& Lyman, R. F. Drosophila bristles and the nature of quantitative genetic variation. Philos. Trans. R. Soc. Lond. B Biol. Sci. 360, 1513-1527 (2005).

10. Rowe S. J., White I. M. S., Avendaño S. \& Hill W. G. Genetic heterogeneity of residual variance in broiler chickens. Genet. Sel. Evol. 38, 617-635 (2006).

11. Harbison, S. T., McCoy, L. J. \& Mackay, T. F. C. Genome-wide association study of sleep in Drosophila melanogaster. BMC Genomics 14, 281 (2013).

12. Garlapow, M. E., Huang, W., Yarboro, M. T., Peterson, K. R. \& Mackay, T. F. C. Quantitative genetics of food intake in Drosophila melanogaster. PLoS One 10, e0138129 (2015)

13. Morgante, F., Sørensen, P., Sorensen, D. A., Maltecca, C. \& Mackay, T. F. C. Genetic architecture of micro-environmental plasticity in Drosophila melanogaster. Sci. Rep. 5, 9785 (2015).

14. Lin, Y., Chen, Z. X., Oliver, B. \& Harbison, S. T. microenvironmental gene expression plasticity among individual Drosophila melanogaster. G3 (Bethesda) 6, 4197-4210 (2016).

15. Wang, J. B., Lu, H. L. \& St Leger, R. J. The genetic basis for variation in resistance to infection in the Drosophila melanogaster genetic reference panel. PLoS Pathog. 13, e1006260 (2017).

16. Hekmat-Scafe, D. S., Scafe, C. R., McKinney, A. J. \& Tanouye, M. A. Genome-wide analysis of the odorant-binding protein gene family in Drosophila melanogaster. Genome Res. 12,1357-1369 (2002). 
512 17. Vieira, F. G., Sánchez-Gracia, A. \& Rozas, J. Comparative genomic analysis of the odorant-binding protein family in 12 Drosophila genomes: purifying selection and birthand-death evolution. Genome Biol. 8, R235 (2007).

18. Larter, N. K., Sun, J. S. \& Carlson, J. R. Organization and function of Drosophila odorant binding proteins. Elife 5, e20242 (2016).

517 19. Findlay G. D., Yi, X., Maccoss, M. J. \& Swanson, W. J. Proteomics reveals novel Drosophila seminal fluid proteins transferred at mating. PLoS Biol. 6, e178 (2008).

519 20. Sun, J. S. et al. Humidity response depends on the small soluble protein Obp59a in Drosophila. eLife 7, e39249 (2018a).

521 21. Johnstun, J. A. et al. Functional diversification, redundancy, and epistasis among paralogs of the Drosophila melanogaster Obp50a-d gene cluster. Mol. Biol. Evol. 38, 2030-2044 (2021).

524 22. Swarup, S., Williams, T. I. \& Anholt, R. R. H. Functional dissection of Odorant binding 525 protein genes in Drosophila melanogaster. Genes Brain Behav. 10, 648-657 (2011).

526 23. Swarup, S., Morozova, T. V., Sridhar, S., Nokes, M. \& Anholt, R. R. H. Modulation of 527 feeding behavior by odorant-binding proteins in Drosophila melanogaster. Chem. Senses 39, 125-132 (2014).

24. Shorter, J. R. et al. Genetic architecture of natural variation in Drosophila melanogaster proteins expressed in gustatory and olfactory sensilla. Genetics 159, 1059-1072 (2001).

533 26. Baker, B. M. et al. The Drosophila brain on cocaine at single-cell resolution. Genome Res. Advance online publication (2021). doi: 10.1101/gr.268037.120.

535 27. Mokashi, S. S. et al. Developmental alcohol exposure in Drosophila: effects on adult phenotypes and gene expression in the brain. Front. Psychiatry in press (2021). doi: 10.3389/fpsyt.2021.699033. 
538

539

540

541

542

543

544

545

546

547

548

549

550

551

552

553

554

555

556

557

558

559

560

561

28. Mackay, T. F. C. et al. The Drosophila melanogaster Genetic Reference Panel. Nature 482, 173-178 (2012).

29. Huang, W. et al. Natural variation in genome architecture among 205 Drosophila melanogaster Genetic Reference Panel lines. Genome Res. 24, 1193-1208 (2014).

30. Morozova, T. V. et al. A Cyclin E centered genetic network contributes to alcoholinduced variation in Drosophila development. G3 (Bethesda) 8, 2643-2653 (2018).

31. Mi, H. \& Thomas, P. PANTHER pathway: an ontology-based pathway database coupled with data analysis tools. Methods Mol. Biol. (Clifton, N.J.) 563, 123-140 (2009).

32. Manolio, T. A. et al. Finding the missing heritability of complex diseases. Nature 461, 747-753 (2009).

33. Visscher, P. M. et al. 10 years of GWAS discovery: Biology, function, and translation. Am. J. Hum. Genet. 101, 5-22 (2017).

34. Yengo, L. et al. Meta-analysis of genome-wide association studies for height and body mass index in $~ 700000$ individuals of European ancestry. Hum. Mol. Genet. 27, 36413649 (2018).

35. Flint, J. \& Mackay, T. F. C. Genetic architecture of quantitative traits in mice, flies, and humans. Genome Res.19, 723-733 (2009).

36. Mackay, T. F. C., Stone, E. A. \& Ayroles, J. F. The genetics of quantitative traits: Challenges and prospects. Nat. Rev. Genet. 10, 565-577 (2009).

37. Mackay, T. F. C. \& Huang, W. Charting the genotype-phenotype map: lessons from the Drosophila melanogaster Genetic Reference Panel. Wiley Interdiscip. Rev. Dev. Biol. 7, 10.1002/wdev.289 (2018).

38. Fisher, R. A. The correlation between relatives on the supposition of Mendelian inheritance. Trans. Roy. Soc. Edin. 52, 399-433 (1918). 
562

563

564

565

566

567

568

569

570

571

572

573

574

575

576

577

578

579

580

581

582

583

584

585

586

587

39. Lai, C., Lyman, R. F., Long, A. D., Langley, C. H. \& Mackay, T. F. C. Naturally occurring variation in bristle number and DNA polymorphisms at the scabrous locus in Drosophila melanogaster. Science 266, 1697-1702 (1994).

40. Huang, W. et al. 2020. Context-dependent genetic architecture of Drosophila life span. PLoS Biology 18, e3000645 (2020).

41. Rautela, I. et al. An extensive review to facilitate understanding of CRISPR technology as a gene editing possibility for enhanced therapeutic applications. Gene 785, 145615 (2021).

42. Zeballos, C. MA. \& Gaj, T. Next-generation CRISPR technologies and their applications in gene and cell therapy. Trends Biotechnol. 39, 692-705 (2021).

43. Rossi, A. et al. Genetic compensation induced by deleterious mutations but not gene knockdowns. Nature 524, 230-233 (2015).

44. El-Brolosy, M. A. \& Stainier, D. Y. R. Genetic compensation: A phenomenon in search of mechanisms. PLoS Genet. 13, e1006780 (2017).

45. Peretz, L. et al. Combined shRNA over CRISPR/cas9 as a methodology to detect offtarget effects and a potential compensatory mechanism. Sci. Rep. 8, 93 (2018).

46. Yamamoto, A., Anholt, R. R. H. \& Mackay, T. F. C. Epistatic interactions attenuate mutations affecting startle behaviour in Drosophila melanogaster. Genet. Res. (Camb). 91, 373-382 (2009).

47. Swarup, S. et al. Extensive epistasis for olfactory behaviour, sleep and waking activity in Drosophila melanogaster. Genet. Res. (Camb.) 94, 9-20 (2012).

48. Greene, C. S., Penrod, N. M., Williams, S. M. \& Moore, J. H. Failure to replicate a genetic association may provide important clues about genetic architecture. PLoS One 4, e5639 (2009).

49. Huang, W. et al. Epistasis dominates the genetic architecture of Drosophila quantitative traits. Proc. Natl. Acad. Sci. U. S. A. 109, 15553-15559 (2012). 
50. Mackay, T. F. C. Epistasis and quantitative traits: using model organisms to study genegene interactions. Nat. Rev. Genet. 15, 22-33 (2014).

51. Magwire, M. M. et al. Quantitative and molecular genetic analyses of mutations increasing Drosophila life span. PLoS Genet. 6, e1001037 (2010).

52. Zwarts, L. et al. Complex genetic architecture of Drosophila aggressive behavior. Proc. Natl. Acad. Sci. U. S. A. 108, 17070-17075 (2011).

53. Sun, J. S., Xiao, S. \& Carlson, J. R. The diverse small proteins called odorant-binding proteins. Open Biol. 8,180208 (2018b).

54. Rihani, K., Ferveur, J. F. \& Briand, L. The 40-year mystery of insect odorant-binding proteins. Biomolecules 11, 509 (2021).

55. Gratz, S. J. et al. Highly specific and efficient CRISPR/Cas9-catalyzed homologydirected repair in Drosophila. Genetics 196, 961-971 (2014).

56. Gratz, S. J., Rubinstein, C. D., Harrison, M. M., Wildonger, J. \& O'Connor-Giles, K. M. CRISPR-Cas9 genome editing in Drosophila. Curr. Prot. Mol. Biol. 111, 31.2.1-31.2.20 (2015).

57. Ja, W. et al. Prandiology of Drosophila and the CAFE assay. Proc. Natl. Acad. Sci. USA, 104, 8253-8256 (2007).

58. Chiu, J. C., Low, K. H., Pike, D. H., Yildirim, E. \& Edery, I. Assaying locomotor activity to study circadian rhythms and sleep parameters in Drosophila. J. Vis. Exp. 43, 2157 (2010).

59. Cichewicz, K. \& Hirsh, J. ShinyR-DAM: a program analyzing Drosophila activity, sleep and circadian rhythms. Commun. Biol. 1, 25 (2018).

60. Morgan, T. J. \& Mackay, T. F. C. Quantitative trait loci for thermotolerance phenotypes in Drosophila melanogaster. Heredity 96, 232-242 (2006).

61. Shaw, P. J., Cirelli, C., Greenspan, R. J. \& Tononi, G. Correlates of sleep and waking in Drosophila melanogaster. Science 287, 1834-1837 (2000). 
614 62. Hendricks, J. C. et al. Rest in Drosophila is a sleep-like state. Neuron 25, 129-138

615

616

617

618

619

620

621

622

623

624

625

626

627

628

629

630

631 (2000).

63. Jensen, K. et al. Purification of transcripts and metabolites from Drosophila heads. J. Vis. Exp. 73, e50245 (2013).

64. Chen, S. et al. AfterQC: automatic filtering, trimming, error removing and quality control for fastq data. BMC Bioinformatics 18, 91-100 (2017).

65. Bushnell, B. BBTools: A suite of fast, multithreaded bioinformatics tools designed for analysis of DNA and RNA sequence data. Joint Genome Institute. Available from: https://jgi.doe.gov/data-and-tools/bbtools/ (2018).

66. Wu, T. D. \& Nacu, S. Fast and SNP-tolerant detection of complex variants and splicing in short reads. Bioinformatics 26, 873-881 (2010).

67. Liao, Y., Smyth, G. K. \& Shi, W. 2013. The Subread aligner: fast, accurate and scalable read mapping by seed-and-vote. Nucleic Acids Res. 41, e108-e108 (2013).

68. Smid, M. et al. 2018. Gene length corrected trimmed mean of M-values (GeTMM) processing of RNA-seq data performs similarly in intersample analyses while improving intrasample comparisons. BMC Bioinformatics 19, 1-13 (2018).

69. Bader, G. D. \& Hogue, C. W. V. An automated method for finding molecular complexes in large protein interaction networks. BMC Bioinformatics 4, 2 (2003). 
632 Table 1. Genotypes used in this study. The control genotype, CSB, has the major allele for all

633 Obp56h DGRP SNPs.

\begin{tabular}{cccccc}
\hline Symbol & Annotation & $\begin{array}{c}\text { Chromosomal } \\
\text { Location } \\
\text { (Flybase } \\
\text { version 6) }\end{array}$ & $\begin{array}{c}\text { DGRP } \\
\text { Minor } \\
\text { Allele }\end{array}$ & $\begin{array}{c}\text { DGRP } \\
\text { Major } \\
\text { Allele }\end{array}$ & $\begin{array}{c}\text { DGRP } \\
\text { Minor } \\
\text { Allele } \\
\text { Frequency }\end{array}$ \\
\hline Obp56h & Obp56h null & & & & \\
Obp56h $h^{A 5510 C}$ & A5510C upstream & $2 R: 19,815,510$ & $\mathrm{C}$ & $\mathrm{A}$ & 0.264 \\
Obp56h $^{\text {T5613C }}$ & T5613C F13L & $2 R: 19,815,613$ & $\mathrm{C}$ & $\mathrm{T}$ & 0.105 \\
Obp56h $h^{\text {C5849G }}$ & C5849G T72S & $2 R: 19,815,849$ & $\mathrm{G}$ & $\mathrm{C}$ & 0.049 \\
Obp56h ${ }^{\text {A6182T }}$ & A6182T 3'UTR & $2 R: 19,816,182$ & $\mathrm{~T}$ & $\mathrm{~A}$ & 0.006 \\
Obp56h $^{\text {T6247A }}$ & T6247A downstream & $2 R: 19,816,247$ & $\mathrm{~A}$ & $\mathrm{~T}$ & 0.158 \\
\hline
\end{tabular}




\section{Figure Legends}

636 Figure 1. Construction of the Obp56h null allele and a series of DGRP minor alleles of Obp56h. Dark gray boxes represent exons of the Obp56h gene and light gray boxes indicate the intron and 5' and 3' untranslated sequences. We designed guide RNAs flanking the Obp56h gene for CRISPR/Cas9-mediated deletion at the cut sites, indicated by the scissor symbols, in the Canton $S-B(C S B)$ genetic background. We replaced the gene with a cassette that contains

642 and with SV40 polyadenylation sequences, loxP sites (blue boxes) for Cre-mediated removal of

643 the insert, and an attP site (purple box) for PhiC31-mediated reinsertion. We then performed 644 PhiC31-mediated site-specific recombination to generate Obp56h alleles with indicated 645 nucleotide substitutions (arrows) that were generated by site directed mutagenesis. The 646 Obp56h alleles are in the CSB background (which has the major allele for each of the five 647 Obp56h SNPs) except for each single substituted base pair and the short 34 bp attL and 60 bp 648 att $R$ sequences (purple boxes) that remained after recombination.

651 Viability. (B) Sex ratio. (C, D) Sucrose consumption. (E, F) Survival under starvation conditions. 652 (G, H) Survival curves under starvation stress. (I, J) Recovery from a chill-induced coma. (K, L) 653 Heat shock survival. For assays where males and females were scored separately, males are 654 indicated in blue females in pink. ${ }^{*}: P<0.05 ;{ }^{* *}: P<0.001$; ${ }^{* \star}: P<0.0001$ (Supplementary 655 Table 3). 
Figure 3. Effects of Obp56h alleles on activity and sleep phenotypes. (A, B) Total activity. pink. ${ }^{*}: P<0.05 ;{ }^{* *}: P<0.001 ;{ }^{* *}: P<0.0001$ (Supplementary Table 3).

660

Figure 4. Summary of RNA sequencing analyses of Obp56h alleles. (A) Average Numbers of differentially expressed genes (FDR < 0.05) for every pairwise comparison of Obp56h alleles. Females (pink) are above the diagonal and males (blue) are below the diagonal.

Figure 5. Protein-protein interaction network from differentially expressed transcripts based on MCODE clustering and the functional annotations of the sub-networks are based on

672 statistically significant (Benjamini-Hochberg FDR < 0.05) enrichment of their Gene Ontology

673 Pathways. The colors of the nodes represent the MCODE connectivity index and the shape of 674 the nodes represents whether they are cluster seeds (squares), in cluster (circles) or 675 unclustered (diamonds). Edges represent known protein-protein interactions. among Obp56h alleles in females. The network was constructed using known interactions

679 from the String database for all significantly (Benjamini-Hochberg FDR $<0.05$ ) differentially 680 expressed transcripts. Genes encoding the transcripts are organized into circular sub-networks 
bioRxiv preprint doi: https://doi org/10.1101/2021.06.28.450219; this version posted June 29, 2021. The copyright holder for this preprint (which was not certified by peer review) is the author/funder, who has granted bioRxiv a license to display the preprint in perpetuity. It is made available under aCC-BY-NC-ND 4.0 International license.

681 based on MCODE clustering and the functional annotations of the sub-networks are based on

682 statistically significant (Benjamini-Hochberg FDR $<0.05$ ) enrichment of their Gene Ontology

683 Pathways. The colors of the nodes represent the MCODE connectivity index and the shape of

684 the nodes represents whether they are cluster seeds (squares), in cluster (circles) or 685 unclustered (diamonds). Edges represent known protein-protein interactions. 


\section{Supplementary Material}

\section{Supplementary Figure 1. Crossing scheme for the generation of the homozygous} Obp56h null allele and DGRP minor alleles for five DGRP SNPs (Obp56hod) in the CSB genetic background. All balancer and marker chromosomes and chromosomes for PhiC31mediated insertion and Cre-mediated deletion were substituted into CSB. $w^{1118}$ denotes the CSB $X$ chromosome. All third chromosomes are from CSB for all genotypes and are not shown.

\section{Supplementary Figure 2. Frequency distributions of $C V$ between replicates for expressed} genes for each genotype. The most extreme $C V$ values are for $C V>1$ (vertical red line). (A) Males. (B) Females.

\section{Supplementary Figure 3. MDS plots of Obp56h alleles based on $\mathrm{CV}$ values from genes} that contribute to micro-environmental plasticity of the transcriptome. Multi-dimensional scaling plots represent the ordination of allelic variants, CSB and the Obp56h null allele based on the $C V$ values for micro-environmental plasticity of the transcriptome. (A) Males. (B) Females. The percent overall variation explained by each axis is represented in the titles of the axes within parentheses.

Supplementary Table 1. LD between Obp56h SNPs in the DGRP. Obp56h ${ }^{A 6182 T}$ is not included since the frequency of the minor allele is too rare to calculate LD in a sample of this size. $r^{2}$ is above the diagonal and $D^{\prime}$ is below the diagonal. All values of LD are significant at $P<$ $0.0001\left(x^{2}{ }_{1}\right.$ goodness of fit test) 

DGRP SNP minor alleles. ANOVA results for all genotypes. (B) Genotype means and significance of individual allele differences from CSB ( $t$-tests).

Supplementary Table 4. Summary of significance of differences of Obp56h alleles from CSB. Entries in each cell are $P$-values. (A) Mean values. (B) Micro-environmental variance heterogeneity. MAF: Minor allele frequency. N/A: Not applicable.

Supplementary Table 5. Comparison of effects and $P$-values for Opb56h alleles in the CSB genetic background and for the same alleles in the DGRP. N/A: effect could not be estimated in the DGRP as the allele was not present in the sample of lines used to quantify chill coma recovery time. Data are from Ref. 11 (total activity) Ref. 29 (starvation survival, chill coma recovery); Ref. 12 (sucrose consumption); and Ref. 30 (viability).

Supplementary Table 6. RNA sequencing raw data. Obp56h alleles are denoted by their

727 superscript. CSB: Canton S B control. M denotes males and F denotes females, and 1 and 2 728 indicate replicates 1 and 2, respectively. (A) Numbers of reads per gene. (B) Filtered and 729 normalized counts/million reads. (C) Conditional means. 
732 with significant (FDR < 0.05) variation among genotypes are shown. (A) Males. (B) Females.

733 DF: Degrees of freedom. SS: Sums of Squares. MS: Mean Squares. F: F ratio statistic. (C)

734 Genes in common between males and females and unique for each sex. (D) Gene set 735 enrichment analyses.

Supplementary Table 8. Significant (FDR < 0.05) differentially expressed genes in pairwise comparisons of Obp56h alleles. $\log _{2} \mathrm{FC}$ is the log2 fold change of allele 2 relative to allele 1. Orange cells denote increased transcript abundance of allele 2 relative to allele 1 , and purple cells represent decreased transcript abundance of allele 2 relative to allele 1. (A) Males. (B) Females.

Supplementary Table 9. Micro-environmental plasticity for gene expression of Obp56h alleles. (A) $C V$ values for co-regulated genes in males. Entries above the threshold of $C V=1$ are in bold font. Expression counts are the medians across all genotypes. P-Values and FDR are from Levene's tests for variance heterogeneity across all genotypes. (B) Co-regulated genes with $C V>1$ for each allele in males. (C) Gene Ontology (GO) enrichment for co-

747 regulated genes with $C V>1$ in males. There was no enrichment for Obp56h ${ }^{5849 G}$ and Obp56h:

748 (D) $C V$ values for co-regulated genes in females. Entries above the threshold of $C V=1$ are in 749 bold font. Expression counts are the medians across all genotypes. P-Values and FDR are from 750 Levene's tests for variance heterogeneity across all genotypes. (E) Co-regulated genes with CV $751>1$ for each allele in females. (F) Gene Ontology (GO) enrichment for co-regulated genes with $752 C V>1$ in females. There was significant enrichment only for Obp56h ${ }^{5849 G}$ and $O b p 56 h^{6182 T}$. 

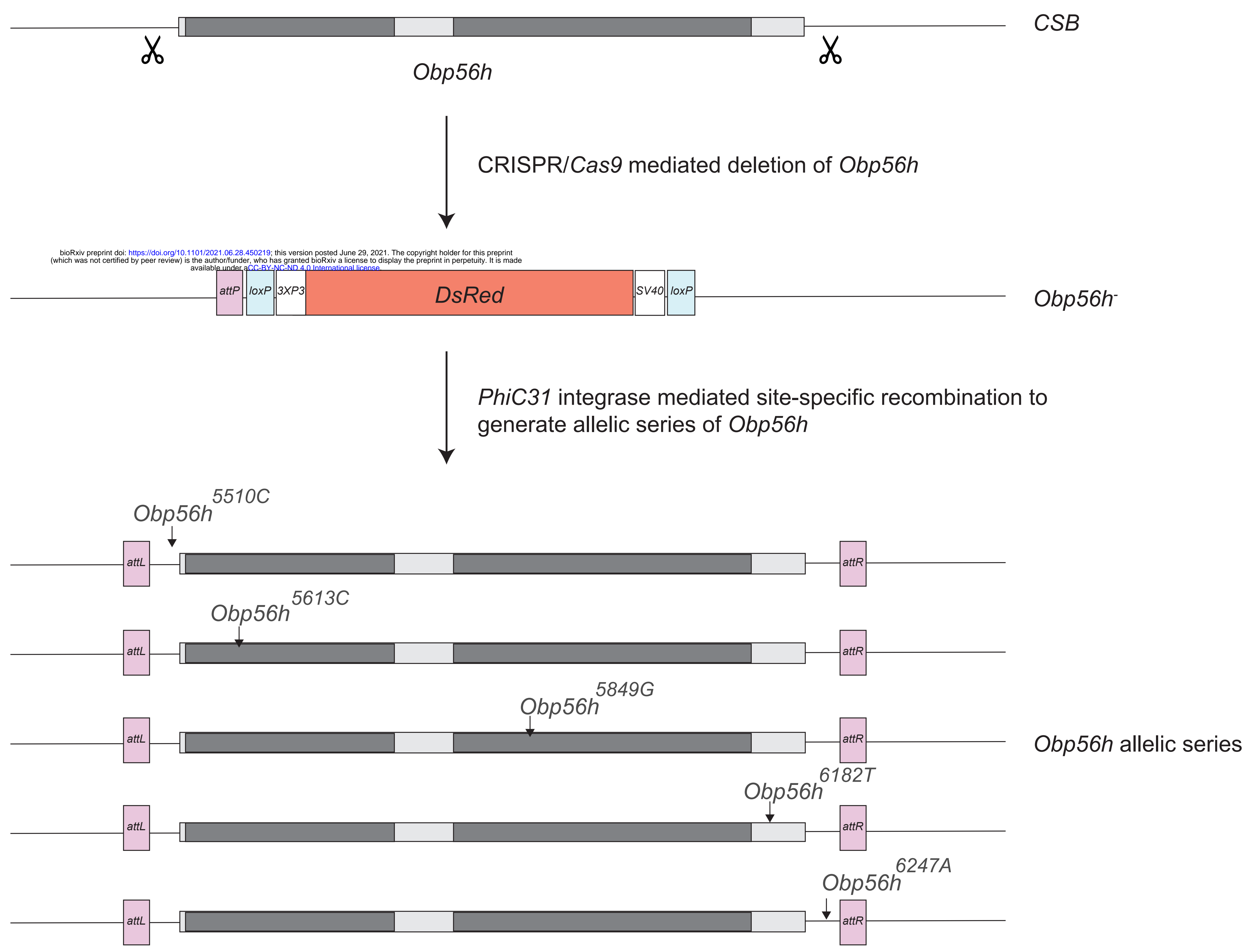


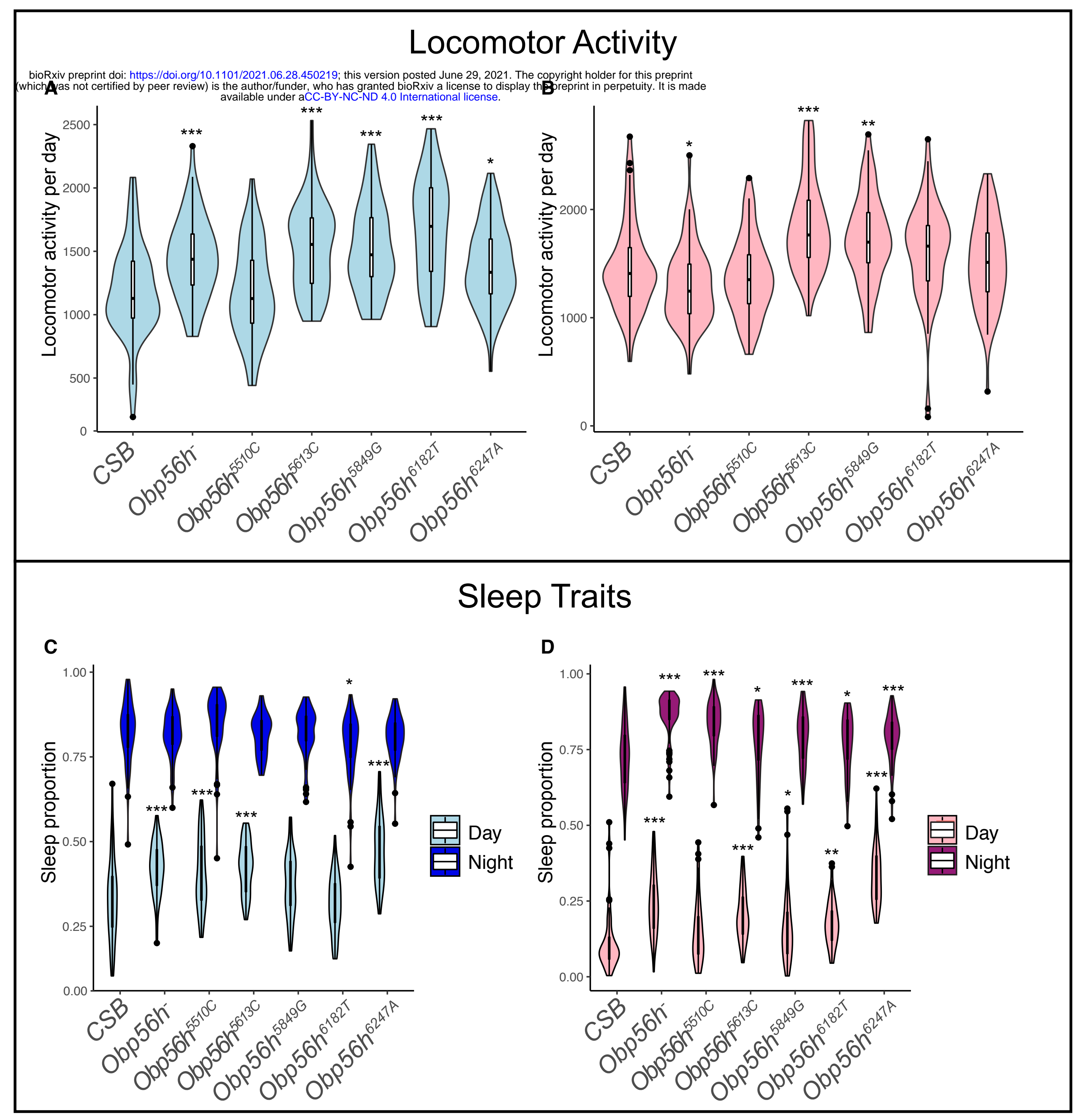


A

\section{Obp56h Expression}

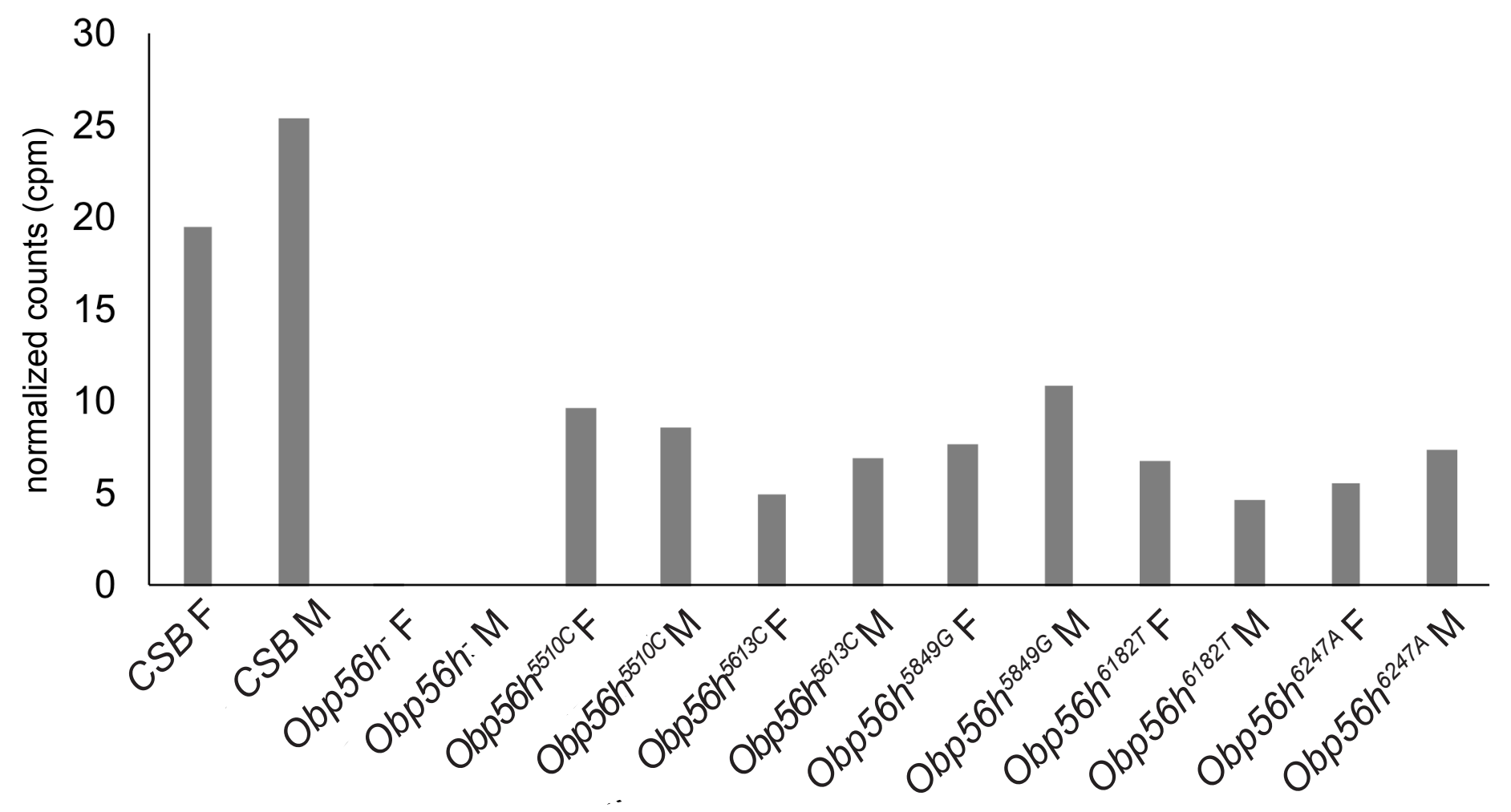

B

Numbers Of Differentially Expressed Genes

CSB

Obp56h ${ }^{-}$

Obp56h $h^{5510 c}$

Obp56h $h^{5613 c}$

Obp56h $h^{5849 G}$

Obp56h ${ }^{6182 T}$

Obp56h ${ }^{6247 A}$

\begin{tabular}{|c|c|c|c|c|c|c|}
\hline 号 & $\begin{array}{l}1 \\
6 \\
10 \\
\stackrel{2}{0} \\
0\end{array}$ & 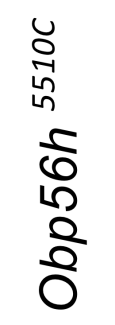 & 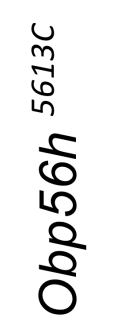 & 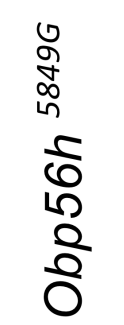 & 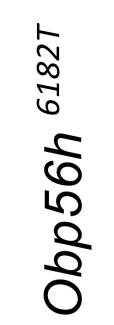 & 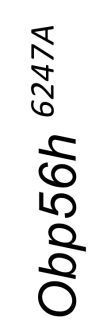 \\
\hline & 66 & 644 & 791 & 61 & 833 & 735 \\
\hline 25 & & 858 & 917 & 22 & 907 & 875 \\
\hline 345 & 443 & & 2 & 857 & 79 & 10 \\
\hline 462 & 508 & 0 & & 895 & 14 & 6 \\
\hline 227 & 218 & 593 & 616 & & 878 & 871 \\
\hline 342 & 399 & 16 & 0 & 390 & & 63 \\
\hline 549 & 578 & 5 & 0 & 645 & 52 & \\
\hline
\end{tabular}



